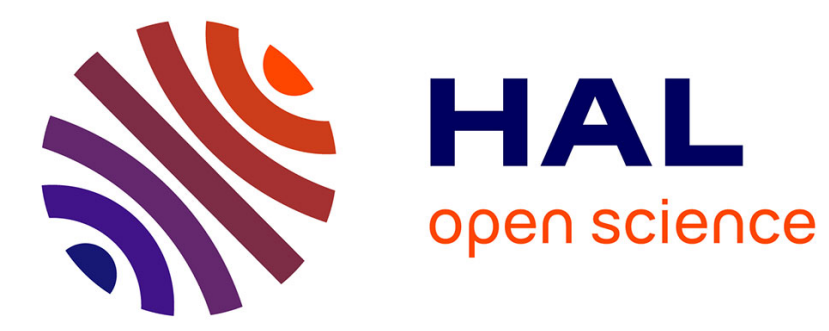

\title{
Tidal constraints on the interior of Venus
}

Caroline Dumoulin, G. Tobie, O. Verhoeven, P. Rosenblatt, N. Rambaux

\section{To cite this version:}

Caroline Dumoulin, G. Tobie, O. Verhoeven, P. Rosenblatt, N. Rambaux. Tidal constraints on the interior of Venus. Journal of Geophysical Research. Planets, 2017, 122 (6), pp.1338-1352. 10.1002/2016JE005249 . hal-02481364

\section{HAL Id: hal-02481364 \\ https://hal.science/hal-02481364}

Submitted on 17 Feb 2020

HAL is a multi-disciplinary open access archive for the deposit and dissemination of scientific research documents, whether they are published or not. The documents may come from teaching and research institutions in France or abroad, or from public or private research centers.
L'archive ouverte pluridisciplinaire HAL, est destinée au dépôt et à la diffusion de documents scientifiques de niveau recherche, publiés ou non, émanant des établissements d'enseignement et de recherche français ou étrangers, des laboratoires publics ou privés. 


\section{Journal of Geophysical Research: Planets}

\section{RESEARCH ARTICLE \\ Tidal constraints on the interior of Venus}

10.1002/2016JE005249

\section{Key Points:}

-We show that viscoelasticity of the mantle strongly increases the potential Love number relative to previous elastic models

- Our results show that a completely solid core cannot be ruled out by the present-day estimation of the potential Love number

- A measure of the tidal lag and a better estimate of the potential Love number are required to constrain Venus' internal structure

Correspondence to:

C. Dumoulin,

caroline.dumoulin@univ-nantes.fr

\section{Citation:}

Dumoulin, C., G. Tobie, O. Verhoeven, P. Rosenblatt, and N. Rambaux (2017), Tidal constraints on the interior of Venus, J. Geophys. Res. Planets, 122, doi:10.1002/2016JE005249.

Received 22 DEC 2016

Accepted 1 JUN 2017

Accepted article online 6 JUN 2017

(C2017. American Geophysical Union. All Rights Reserved.

\section{Introduction} 1995; Mocquet et al., 2011; Aitta, 2012]. state of the core.

\author{
C. Dumoulin' ${ }^{1}$, G. Tobie ${ }^{1}$ (D) , O. Verhoeven ${ }^{1}$ (D), P. Rosenblatt ${ }^{2}$, and N. Rambaux ${ }^{3}$ iD \\ ${ }^{1}$ Laboratoire de Planétologie et Géodynamique, UMR-CNRS 6112, Université de Nantes, Nantes, France, ${ }^{2}$ Royal Observatory \\ of Brussels, Uccle, Belgium, ${ }^{3}$ Institut de Mécanique Céleste et Calcul des Ephémérides, Université Pierre et Marie Curie, \\ Paris, France
}

\begin{abstract}
As a prospective study for a future exploration of Venus, we compute the tidal response of Venus' interior assuming various mantle compositions and temperature profiles representative of different scenarios of Venus' formation and evolution. The mantle density and seismic velocities are modeled from thermodynamical equilibria of mantle minerals and used to predict the moment of inertia, Love numbers, and tide-induced phase lag characterizing the signature of the internal structure in the gravity field. The viscoelasticity of the mantle is parameterized using an Andrade rheology. From the models considered here, the moment of inertia lies in the range of 0.327 to 0.342 , corresponding to a core radius of 2900 to $3450 \mathrm{~km}$. Viscoelasticity of the mantle strongly increases the potential Love number relative to previously published elastic models. Due to the anelasticity effects, we show that the possibility of a completely solid metal core inside Venus cannot be ruled out based on the available estimate of $k_{2}$ from the Magellan mission (Konopliv and Yoder, 1996). A Love number $k_{2}$ lower than 0.27 would indicate the presence of a fully solid iron core, while for larger values, solutions with an entirely or partially liquid core are possible. Precise determination of the Love numbers, $k_{2}$ and $h_{2}$, together with an estimate of the tidal phase lag, are required to determine the state and size of the core, as well as the composition and viscosity of the mantle.
\end{abstract}

Due to similar mass and radius, Venus is usually considered as Earth's twin sister. However, these two planets have followed very different geological and climate evolutions [e.g., Phillips et al., 2001; Noack et al., 2012; Gillmann and Tackley, 2014]. This divergence initiates probably very early during their history and likely reflects significant difference in interior composition and rheology. Our knowledge on the internal structure of Venus relies on a limited set of data: mass, radius, gravity, and topography data, as well as surface temperature and composition at specific locations [e.g., Sohl and Schubert, 2015]. Due to a slow rotation, the hydrostatic oblateness is very small: the observed gravity coefficient $J_{2}$ is 25 times larger than the hydrostatic one, which prevents any estimation of the mean moment of inertia from the sole gravity field measurements. In the absence of data to constrain Venus' density profile, the interior structure of Venus is often a rescaled version of the Earth using the one-dimensional seismological Preliminary Reference Earth Model (PREM, [Dziewonski and Anderson, 1981]) slightly modified to account for Venus' radius and mass [see, e.g., Zharkov, 1983; Yoder,

The only direct existing constraint on the interior structure is provided by the potential Love number estimated from Doppler tracking of Magellan and Pioneer Venus Orbiter $\left(k_{2}=0.295 \pm 0.066\right.$ [Konopliv and Yoder, 1996]). However, the significant error bar prevents any inference of the density profile. Assuming an elastic formulation of the body response of Venus to solar tides for a suite of pressure-scaled versions of the PREM model, Yoder [1995] showed that there is a significant increase in $k_{2}$ between models with a solid core $\left(k_{2} \simeq 0.17\right)$ and models with a liquid core $\left(0.23<k_{2}<0.29\right)$. The value inferred by Konopliv and Yoder [1996] was then considered as evidence for a liquid core. Unfortunately, there are no other observations that can support or deny this conclusion. The absence of an internal magnetic field solely indicates that the cooling of the core is not compatible with an Earth-like chemically driven dynamo [Stevenson et al., 1983]. Constraining the present-day state of the core is essential as it provides constraints on the efficiency of heat extraction by the mantle through Venus' history, notably on the possible cessation of a core dynamo [Stevenson, 2003; Armann and Tackley, 2012]. The conclusions of Yoder [1995] and Konopliv and Yoder [1996] are, however, questionable as they relied on an elastic formulation of the tidal deformation. For a tidal period of 58.4 days, anelasticity is expected to play a significant role [Benjamin et al., 2006] to modify the conclusion regarding the 
Future spacecraft missions to Venus currently under consideration by ESA (EnVision [Ghail et al., 2016]) would offer the opportunity to significantly improve our knowledge of the gravity field from Doppler tracking data relative to the Magellan and Pioneer missions. They should be able to reduce the error bars on the potential Love number, $k_{2}$, and to provide first estimates of tide-induced surface displacement. Moreover, the measurements performed by these missions may provide estimates of the tidal phase lag, thus providing insights on the viscous response of the interior. In this context, it is important to refine the prediction of the tidal response of Venus' interior and to determine how the composition and viscosity of the interior influence the amplitude and lag of the tidal response. Constraining simultaneously the density and viscosity profiles will provide crucial information on the internal dynamics and past evolution of Venus.

In the present study, we compute the viscoelastic tidal response of Venus by considering various composition, temperature, and viscosity profiles for the interior. For the composition, we use different end-member models based on different scenarios for its accretion [Basaltic Volcanism Study Project, 1981; Goettel et al., 1981] and combine these with constraints for the surface analysis from Venera and Vega space probes [see Fegley, 2014]. The end-member temperature profiles are defined using published models on Venus' evolution [Steinberger et al., 2010; Armann and Tackley, 2012]. The elastic properties are then computed using the mineralogy derived for each composition model and the assumed temperature profile using the method Perple_ $X$ [Connolly, 2005]. We assume constant viscosity in the silicate mantle and solid part of the core, using values representative of the Earth's mantle and inner core. In section 2, we review and define the interior models considered in the present study. Section 3 describes the employed viscoelastic model including the influence of the massive atmosphere and present a validation of our approach using the Earth's case. In section 4, we present predicted Love number and potential tidal phase lags for the variety of interior models introduced previously. Particular attention is paid to the state of the metallic core. Finally, section 5 provides a discussion of the implications for future spacecraft missions in orbit around Venus and is followed by a brief conclusion.

\section{Mantle Composition and Core State}

\subsection{Geochemical Models}

According to various formation scenarios, distinct composition models based on cosmochemical assumptions and trends among Earth-like planets have been proposed to model Venus' interior (see Basaltic Volcanism Study Project [1981], Anderson [1989], and Fegley [2014] for a review). Their corresponding weight fractions of oxides and modal mineralogies are given in Table 1, along with the Earth pyrolitic composition shown for comparison. The model V1 is the equilibrium condensation model based on temperature- and pressure-dependent thermodynamic equilibrium in a solar nebula model with an adiabatic $P, T$ gradient chosen to provide mineral assemblages at the position of the planets for which densities correspond to the observed density of the planets [Lewis, 1972]. The model V2 is an equilibrium condensation model modified by the use of expanded feeding zones as a result of growing mass of the planet during accretion [Weidenschilling, 1976]. The model V3 presents a composition for Venus based on common fractionation processes in the solar nebula between Earth-like planets and chondrites [Morgan and Anders, 1980]. Morgan and Anders [1980] divided elements into five groups according to similar cosmochemical properties and identified in each group a key element whose abundance can be constrained by geophysical or geochemical data. Five constraints suffice then to define the composition of Venus: mass of the core, abundance of $\mathrm{U}$, and the ratios $\mathrm{K} / \mathrm{U}, \mathrm{Tl} / \mathrm{U}$, and $\mathrm{FeO} /(\mathrm{FeO}+\mathrm{MgO})$. For Venus, the mass of the core is estimated from bulk density using geochemical and geophysical modeling, whereas the abundance of $U$ is based on the apparent trend of $U$ content with size among Earth-like planets. The $\mathrm{K} / \mathrm{U}$ ratio is obtained from $\gamma$ ray spectrometry performed by the spacecraft Venera 8, 9, and 10, whereas the TI/U is chosen to be Earth-like. Among the five constraints, the $\mathrm{FeO} /(\mathrm{FeO}+\mathrm{MgO})$ ratio is probably the least constrained. Morgan and Anders [1980] propose to estimate it from a trend of $\mathrm{FeO}$ content with heliocentric distance observed from spectrophotometric measurement for Mercury, measurements in chondrites, and geophysical estimations for the Earth. The model V4 is based on Ringwood's model [Ringwood, 1977] for planet formation which involves mixtures of low-temperature condensates similar to $\mathrm{C} 1$ chondrites and high-temperature, reduced, metal-rich, devolatized condensates that equilibrated with the nebula in different temperature ranges. A fraction of about $20 \%$ low-temperature condensates can indeed explain the $5 \%$ difference in density between the Earth and Venus by a higher stage of oxidation of Venus compared to the Earth. The model V5 is characterized by a lower Fe content, supposed to explain the $1.9 \%$ observed lower density of Venus compared to the density of a Venus made of the same material as Earth [Basaltic Volcanism Study Project, 1981]. 


\begin{tabular}{|c|c|c|c|c|c|c|}
\hline & \multirow{2}{*}{$\frac{\text { Earth }}{\text { Pyrolite }^{\mathrm{a}}}$} & \multicolumn{5}{|c|}{ Venus } \\
\hline & & $\mathrm{V} 1^{b}$ & $\mathrm{~V} 2^{\mathrm{b}}$ & $\mathrm{V}^{\mathrm{c}}$ & $\mathrm{V} 4^{\mathrm{c}}$ & $\mathrm{V}^{\mathrm{b}}$ \\
\hline $\mathrm{CaO}$ & 3.5 & 3.6 & 3.6 & 3.3 & 3.4 & 3.2 \\
\hline $\mathrm{FeO}$ & 8.36 & 0.24 & 2.1 & 5.4 & 18.7 & 8.1 \\
\hline $\mathrm{MgO}$ & 37.97 & 37.6 & 38.3 & 35.5 & 33.3 & 38.0 \\
\hline $\mathrm{Al}_{2} \mathrm{O}_{3}$ & 4.31 & 3.8 & 3.9 & 4.1 & 3.4 & 3.9 \\
\hline $\mathrm{SiO}_{2}$ & 44.5 & 52.9 & 53.9 & 49.8 & 40.4 & 45.9 \\
\hline
\end{tabular}

\subsection{Venus Temperature Profile}

Among published temperature profiles for Venus' interior, two end-member temperature profiles are chosen: the colder one is derived from Steinberger et al. [2010] (it will be referred to as $T_{\text {cold }}$ in the following) and the hotter one from Armann and Tackley [2012] (it will be referred to as $T_{\text {hot }}$ in the following). These two temperature profiles are represented in Figure 1. The temperature of Steinberger et al. [2010] corresponds to an adiabatic profile rescaled to Venus' pressure range. They used Earth's estimations for the thickness of the hot thermal boundary layer and for the core-mantle boundary temperature. The temperature profile of Armann and Tackley [2012] results from thermochemical evolution models for a basalt/harzburgite composition constrained by present-day topography and geoid and the recent resurfacing history of Venus.

\subsection{Computation of Synthetic Geophysical Data}

Radial density $\rho$ and seismic velocities $v_{p}$ and $v_{S}$ are computed from hydrostatic pressure, temperature, and composition using the Perple_X program [Connolly, 2005] developed by James Connolly (http://www.perplex. ethz.ch) in the mantle and from PREM extrapolation in the metallic core [Dziewonski and Anderson, 1981]. The Perple_X method computes phase equilibria and uses the thermodynamics of mantle minerals developed by Stixrude and Lithgow-Bertelloni [2011]. Although the geochemical models of Venus' interior shown in Table 1 provide specific core compositions with different light elements such as sulfur, oxygen or both, we choose to compute radial density and $P$ wave seismic velocities in the core from PREM polynomial fits rescaled to Venus pressure thus assuming an Earth-like composition of the core. The effect of core composition on the tidal response is expected to be small compared to the effect of the mantle, justifying this assumption. Compared to the mantle, reliable core modeling is also much more difficult to achieve as the accurate determination of physical properties of iron alloys is challenging at core pressure (see, e.g., Morard et al. [2014] for a recent review of binary phase diagrams relevant to Earth's core), although some measurements of density and sound velocity of liquid Fe-Ni with light elements have been recently obtained by shock wave experiments at the Earth's core conditions [see, e.g., Zhang et al., 2016].

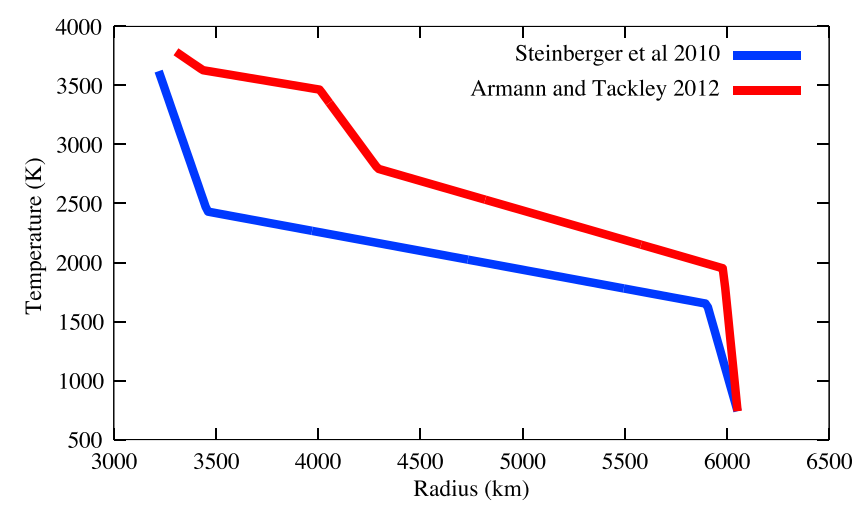

Figure 1. Temperature profiles from Steinberger et al. [2010] (blue, $T_{\text {cold }}$ ) and Armann and Tackley [2012] (red, $T_{\text {hot }}$ ) used to model Venus' interior. 

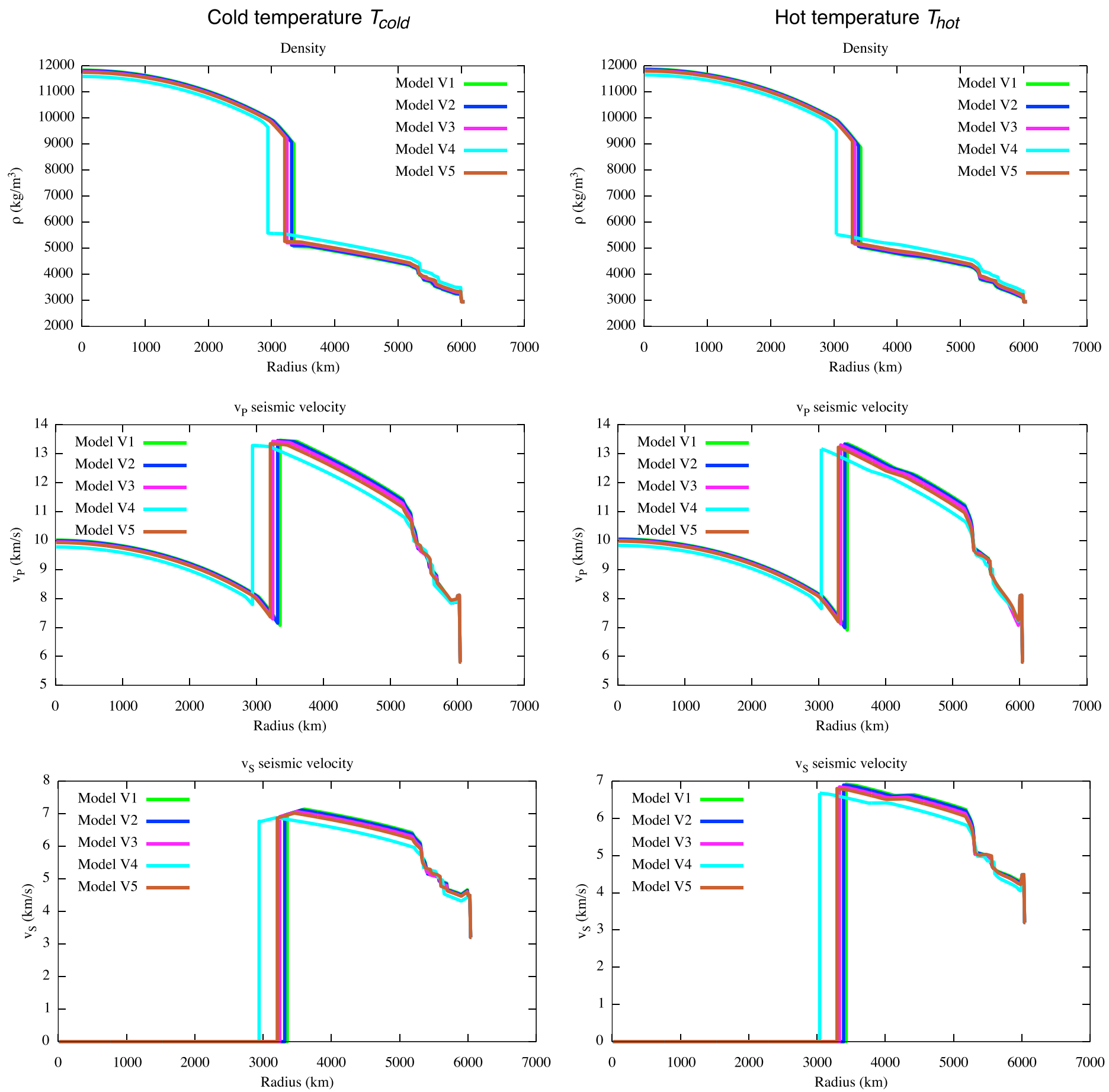

Figure 2. Density $\rho$ and seismic velocities $v_{P}$ and $v_{S}$ corresponding to composition models of Table 1. (left column) Geophysical data associated with the cold temperature profile of Steinberger et al. [2010] and (right column) data associated with the hot temperature profile of Armann and Tackley [2012].

The core-mantle boundary is computed in order to satisfy the mass condition $\left(M_{V}=4.8669 .10^{24} \mathrm{~kg}\right.$ [Steinberger et al., 2010]), and we modify the size of the adiabatic region in the mantle accordingly. The five composition models (presented in section 2.1), along with two extreme temperature profiles in the mantle (see previous section), are associated with this rescaled PREM core model to provide 10 sets of geophysical data. A crust of $2950 \mathrm{~kg} / \mathrm{m}^{3}$ density and $60 \mathrm{~km}$ thickness (as in Steinberger et al. [2010]) is added to each model, with crustal seismic velocities equal to pressure-rescaled PREM (without oceans) values. Density $\rho$ and seismic velocities $v_{P}$ and $v_{S}$ profiles for these 10 models of 500 layers of constant thickness are shown in Figure 2. 
Table 2. Core-Mantle Boundary Radius, Moment of Inertia Factor, Relative Mantle and Core Masses, Pressure and Temperature at the Core-Mantle Boundary (CMB) and Pressure at the Center for Selected Venus Composition Models (See Text) of Table 1 and Temperature Profiles of Steinberger et al. [2010] ( $\left.T_{\text {cold }}\right)$ and Armann and Tackley [2012] $\left(T_{\text {hot }}\right)^{\mathrm{a}}$

\begin{tabular}{|c|c|c|c|c|c|c|c|}
\hline & \multicolumn{3}{|c|}{ Cold Temperature Profile $T_{\text {cold }}$} & \multicolumn{3}{|c|}{ Hot Temperature Profile $T_{\text {hot }}$} & \multirow{2}{*}{$\begin{array}{c}\text { Aitta [2012] } \\
\rho(P) \text { in Earth's Mantle }\end{array}$} \\
\hline & V1 & V4 & V5 & V1 & V4 & V5 & \\
\hline$R_{c}(\mathrm{~km})$ & 3353 & 2941 & 3208 & 3425 & 3038 & 3292 & 3228 \\
\hline$\frac{1}{M R^{2}}$ & 0.330 & 0.342 & 0.334 & 0.327 & 0.339 & 0.331 & 0.338 \\
\hline$M_{\text {mantle+crust }} / M_{\text {Venus }}$ & 0.665 & 0.771 & 0.705 & 0.644 & 0.748 & 0.682 & - \\
\hline$M_{\text {core }} / M_{\text {Venus }}$ & 0.335 & 0.229 & 0.295 & 0.356 & 0.252 & 0.318 & - \\
\hline$P_{\mathrm{CMB}}(\mathrm{GPa})$ & 107 & 127 & 114 & 103 & 123 & 110 & 114 \\
\hline$T_{\mathrm{CMB}}(\mathrm{K})$ & 3636 & 3767 & 3682 & 3733 & 3927 & 3800 & 3630 \\
\hline$P_{\text {center }}(\mathrm{GPa})$ & 295 & 271 & 287 & 299 & 277 & 292 & 274 \\
\hline
\end{tabular}

a Last column shows Aitta [2012]'s model for comparison.

\subsection{Comparison of the Models: Implications for Core Radius and Moment of Inertia Factor}

As core modeling is identical to PREM for each set of data, differences in geophysical data in the core are directly related to differences in the core pressure profile associated with the core size. The signatures of the 10 proposed models are therefore best identified in the mantle density and seismic velocities and in the core size. As shown in Figure 2, there are only small differences in density and seismic velocities among the five geochemical models for a given temperature profile. Nevertheless, the model V4, characterized by a high $\mathrm{FeO}$ content, presents a 200 to $300 \mathrm{~kg} / \mathrm{m}^{3}$ higher mantle density than the other models, resulting in a 300 to $400 \mathrm{~km}$ smaller core radius and a $3 \%$ higher moment of inertia (see Table 2). The moment of inertia factor is computed using the radial density structures inferred for each composition and temperature profile. The value of the moment of inertia factor classically mentioned in the literature for Venus, and obtained considering a scaled version of the Earth structure, is 0.334 [e.g., Zharkov, 1983]. It corresponds to the value computed for the Earth-like pyrolitic model (V5), using the cold temperature profile. For a given composition, the $600 \mathrm{~K}$ difference in the two temperature profiles at $1500 \mathrm{~km}$ depth induces differences in mantle density and seismic velocities of the order of $50 \mathrm{~kg} / \mathrm{m}^{3}$ and $0.2 \mathrm{~km} / \mathrm{s}$, respectively. The temperature increase from the profile of Steinberger et al. [2010] to the profile of Armann and Tackley [2012] induces therefore an average 1\% decrease in mantle density, resulting in a $100 \mathrm{~km}$ larger core radius and a $1 \%$ smaller value for the moment of inertia. Values of the moment of inertia factor reported in Table 2 sample quite well (and extend slightly) the $[0.331,0.341]$ range, obtained by Yoder [1995]. None of these models presents a pressure at the center greater than $300 \mathrm{GPa}$, which prevents the existence of a solid inner core for our assumed PREM core modeling. Each model is defined by different temperature or composition, leading to different phase transitions and therefore different pressure profiles. The pressure at the core-mantle boundary and at the center lies in the range [103, 127] GPa and [271, 299] GPa, respectively. Such ranges include the model proposed by Aitta [2012]. In his study, Aitta [2012] proposed an internal structure of Venus assuming that the variation of density with pressure is identical in Venus' mantle and in the Earth's one (therefore, composition and temperature are supposed to be similar in both planetary mantles). She used the theory of tricritical phenomena to estimate the density variations in the core, assuming that it is entirely liquid.

Among the five composition models, models V1 and V4 may be considered as end-members as they are characterized by the largest difference in the density and seismic velocity profiles, resulting to the highest variability in core radius and moment of inertia. We then chose these two compositional models together with the model V5 which is the closest to an Earth-like Venus to investigate the body response of Venus to solar tides. These selected models will be referred to using both the compositional model (V1, V4, or V5) and the temperature profile ("cold," i.e., Steinberger et al. [2010], $T_{\text {cold }}$, and "hot," i.e., Armann and Tackley [2012], $T_{\text {hot }}$ ).

\section{Computation of Tidal Deformation}

\subsection{Description of the Viscoelastic Model}

The viscoelastic deformation of Venus under the action of periodic tidal forces is computed following the method of Tobie et al. [2005]. Venus is assumed to be divided into an inviscid fluid iron core and a viscoelastic solid mantle. The density profile and elastic properties in the iron core and mantle are computed 
using the method provided in section 2.3. From these profiles, the Poisson equation and the equation of motions are solved for small perturbations in the frequency domain assuming a compressible Andrade rheology [Castillo-Rogez et al., 2011]. The complex compliance, which defines the complex shear modulus, for an Andrade model is given by

$$
J(\chi)=\frac{1}{\chi}-\frac{i}{\eta \chi}+\beta(i \chi)^{-\alpha} \Gamma(1+\alpha),
$$

where $\eta$ is the viscosity; $\chi$ is the tidal frequency $(\chi=2(\omega-n)) ; \omega$ and $n$ are the spin rate and mean orbital motion, respectively; and $\alpha$ and $\beta$ are parameters describing the frequency dependence and the amplitude of the transient response, respectively. Comparison with available experimental data for rock and applications to the Earth (see section 3.3) indicates that the Andrade model is a good approximation to describe the anelastic attenuation at tidal frequencies [Castillo-Rogez et al., 2011]. For the $\alpha$ parameter, we explore a range of values varying between 0.2 and 0.3 , which frames the typical value required to explain the $Q$ factor of the Earth's mantle (see section 3.3). For the $\beta$ parameter, following the approximation of Castillo-Rogez et al. [2011], we assume that $\beta \simeq \mu^{\alpha-1} \eta^{-\alpha}$, where $\mu$ is the shear modulus $\left(\mu=\rho v_{s}^{2}\right)$, which is justified for olivine [Tan et al., 2001; Jackson et al., 2002].

The Love numbers, $k_{2}$ and $h_{2}$, characterizing the potential perturbation and surface radial displacement, respectively, and the dissipation function, $Q^{-1}$, are computed by integrating the radial functions associated with the radial and tangential displacements $\left(y_{1}\right.$ and $y_{3}$, respectively), the radial and tangential stresses $\left(y_{2}\right.$ and $\left.y_{4}\right)$, and the gravitational potential $\left(y_{5}\right)$, as defined by Takeushi and Saito [1972]. The deformation of the liquid core and of the dense atmospheric layer is assumed to be static, and the simplified formulation of Saito [1974] relying on to radial functions, $y_{5}$ and $y_{7}$, is thus employed. The solution in the solid part of the interior is expressed as the linear combination of three independent solutions, which reduces to two independent solutions in the fluid part. The system of six differential equations is solved by integrating the three independent solutions using a fifth-order Runge-Kutta method with adjustive stepsize control from the center $(r=0 \mathrm{~km})$ to the upper atmosphere $\left(r=R_{p}+100=R_{\mathrm{atm}} \mathrm{km}\right.$ ) and by applying the appropriate boundary conditions (see, for more details, Takeushi and Saito [1972], Saito [1974], and Tobie et al. [2005]). The complex Love numbers, $k_{2}^{*}$ and $h_{2}^{*}$, are determined from the radial functions, $y_{5}\left(R_{P}\right)$ and $y_{1}\left(R_{P}\right) \mathrm{m}$, respectively, at Venus' surface $\left(r=R_{p}\right)$, and the global dissipation function, $Q^{-1}$, by the ratio between the imaginary part and the module of $k_{2}^{*}$ :

$$
k_{2}^{*}=y_{5}\left(R_{P}\right)-1 ; h_{2}^{*}=y_{1}\left(R_{P}\right) g\left(R_{P}\right) ; Q^{-1}=\mathfrak{J}\left(k_{2}^{*}\right) /\left\|k_{2}^{*}\right\| .
$$

\subsection{Viscosity Profiles in the Mantle}

We investigate a large range of mantle viscosities: from $10^{19}$ to $10^{22} \mathrm{~Pa}$ s. Viscosity in the mantle is supposed to vary with temperature and pressure following an Arrhenius law and with grain size or strain rate, depending on the dominant deformation mechanism (diffusion or dislocation creep, see Karato [2008] for details). For the Earth, values of activation energy and volume are obtained from laboratory measurements [Karato and $W u$, 1993]. Diffusion creep is often considered to be dominant in the Earth's lower mantle [Karato and Wu, 1993], leading to a strong influence of the grain size (that can vary from $0.1 \mathrm{~mm}$ to few millimeters) on the absolute value of the viscosities (several orders of magnitude). Morever, the grain size is probably not uniform in the a silicate mantle, since it varies with the initial grain size, time, and activation enthalpy for grain growth [Korenaga, 2005]. The grain size then depends on the dynamic history (mixing and dynamic recrystallization) and therefore on the thermal evolution of the mantle [Solomatov and Reese, 2008]. In turn, the grain size is directly related to the stress state, influencing the style of convection [Rozel, 2012]. Deriving a unique viscosity profile from pressure and temperature profiles is therefore not possible without several assumptions that vary with the studies [Armann and Tackley, 2012; Steinberger et al., 2010].

Another possibility to investigate the viscosity structure of a planetary mantle is to analyze the geoid and topography at different degrees (see Richards and Hager [1984], for instance, for an Earth's study). This approach has been performed on Venus by Pauer et al. [2006]. They obtain families of viscosity profiles, considering three to five layers (note that even constant viscosity beneath a pronounced lithosphere is a plausible configuration). However, this method provides the viscosity contrasts that best explain the data but not the absolute values. On Earth, absolute values for viscosities are mainly derived from postglacial rebound data [e.g., Mitrovica and Forte, 2004], a method that cannot be applied on another planet, lacking of time-varying measures of topography. 
The structure and absolute value of viscosity in Venus' mantle being therefore largely unknown, we prefer to consider in our study a constant viscosity representative of the averaged profile. We nevertheless compare our constant viscosity case with two variable viscosity profiles in order to evaluate its effect on the tidal deformation. We then computed the tidal deformation obtained for model V5- $T_{\text {hot }}$ using a viscosity profile obtained with the following equation:

$$
\eta=\frac{1}{2} A_{0}^{-1} d^{2.5} \exp \frac{E_{a}+P . V_{a}}{R T},
$$

using the dry olivine parameters $\left(E_{a}=300 \mathrm{~kJ} / \mathrm{mol}, V_{a}=6 \mathrm{~cm}^{3} / \mathrm{mol}\right.$, and $\left.A_{0}=6.08 \times 10^{-19} \mathrm{~Pa}^{-1} \mathrm{~s}^{-1}\right)$ and a grain size $d$ equal to 0.1 or $1 \mathrm{~mm}$. We compared the results to the tidal deformation obtained for a constant mantle viscosity (set to the average of the radially varying profile, i.e., $1.73 \times 10^{19} \mathrm{~Pa}$ s for a grain size of $0.1 \mathrm{~mm}$ and $5.49 \times 10^{21}$ Pa s for $1 \mathrm{~mm}$ ). We performed a third test, using the best fit four-layer viscosity structure from Pauer et al. [2006], setting the absolute values of viscosity to obtain the same radially average viscosity as mentioned above. The largest difference on $k_{2}$ is of $2.1 \%$ (comparing the three models with the smallest averaged viscosity) and of $1.8 \%$ (for the largest). This difference is negligible compared to the uncertainty range for measured $k_{2}$ (more than 22\%). However, the largest difference on the dissipation factor $Q$ is higher. It increases with the averaged value of the viscosity: $3.3 \%$ for the lowest one and $14.8 \%$ for the largest. Such differences are smaller than the uncertainty bars on $Q$ due to the rheology dependence on the parameter alpha (see section 3.1) which are increasing a lot with the mantle viscosity: up to $30 \%$ for a viscosity of $10^{22}$ Pas (see section 4 and Figure 4). We therefore consider that our approach using a constant viscosity in the mantle is a good approximation at first order.

\subsection{Validation of the Model: The Earth's Case}

In this section, we use the Earth as a test case to validate our modeling approach. We compute geophysical data for the Earth using the same procedure as presented above (sections 2.1 and 2.3). The Perple_X algorithm was used to compute density $\rho$ and seismic velocities $v_{P}$ and $v_{S}$ for a terrestrial mantle with pyrolite composition [Irifune, 1987] (see Table 1) associated with Stacey and Davis [1998]'s geotherm. The pressure was derived from the density profile assuming hydrostatic conditions. The model was composed of 500 layers of constant thickness. Crust and core data were computed as pressure-rescaled PREM (without the ocean) values. Crustal thickness was constrained to be $24 \mathrm{~km}$, whereas the core-mantle boundary was tuned to fit the global mass value (as in section 2.3), resulting in a $1 \mathrm{~km}$ smaller core radius compared to the PREM estimate. The strong similarity between our model and PREM data for the Earth (a maximum of $2 \%$ of difference on $\rho, v_{p}$, and $v_{s}$ ) allows us to confidently use the Perple_X algorithm to compute Venus mantle geophysical data. This small discrepancy is related to the choice for parameter values governing our model such as the selected reference geotherm and the thermodynamic constants used to compute phase equilibria along with the number and size of the layers chosen to sample Earth's interior.

To compute the viscoelastic response, we assume constant viscosity values in the upper and lower mantles, ranging between $10^{20}-10^{21} \mathrm{~Pa}$ s and $10^{22}-10^{23} \mathrm{~Pa}$, respectively, consistent with the typical viscosity values derived from geophysical constraints [see Čižková et al., 2012, and references therein]. For this range of viscosity and a parameter $\alpha$ between 0.2 and 0.3 , we obtain a potential Love number for a semidiurnal tidal period between 0.303 and 0.304 , which is consistent with the observed value estimated between 0.304 and 0.312 [Ray et al., 2001]. The modeled $Q$ value is very sensitive to the assumed viscosity and parameter $\alpha$. At the period of the semidiurnal tide $\left(M_{2}\right)$, for assumed viscosity ranges, the Earth's $Q$ value of about 280 inferred from satellite tracking and altimetry [Ray et al., 2001] can be reproduced for $\alpha$ values between 0.23 and 0.28 . For this range of $\alpha$, we can see also that we reasonably reproduce the frequency dependence of the Earth's $Q$ inferred from the fortnightly $M_{f}$ tide (13.66 days [Ray and Egbert, 2012]), the Chandler Wooble (433 days [Furuya and Chao, 1996; Benjamin et al., 2006]) and the 18.6 year tide [Benjamin et al., 2006].

\subsection{Venus' Atmosphere}

We also test the effect of a thick atmosphere on the computation of the tidal response of Venus. The atmospheric density profile in the 100 lower kilometers is taken from Steiff et al. [1985] (the Venus International Reference Atmosphere, i.e., VIRA, model). The sound speed is computed using the temperature given by the VIRA model using the following relationship: $c=\left(\gamma R^{*} T\right)^{1 / 2} \cdot R^{*}$ is the gas constant for Venus and is taken to be equal to $191.4 \mathrm{~J} / \mathrm{kg} / \mathrm{K}\left(R^{*}=R / M, M\right.$ being the atmospheric mean molecular weight and $R$ the universal gas constant); $\gamma$ is the adiabatic index and is taken to be equal to 1.29 [cf. Steiff et al., 1985]. Comparison between 


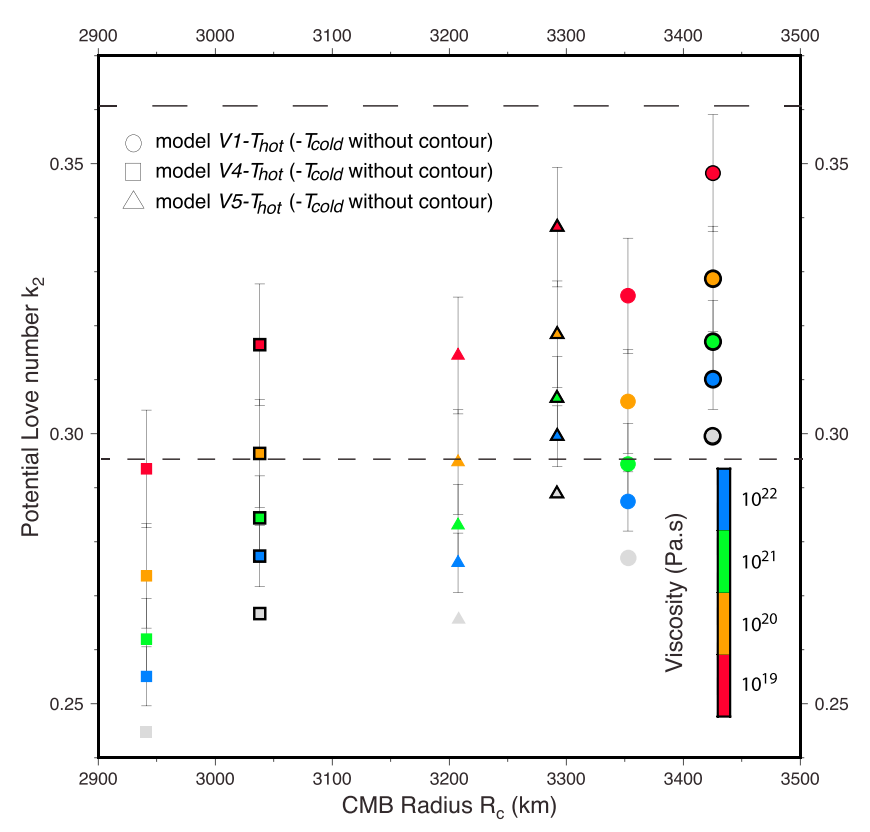

Figure 3. Potential Love number, $k_{2}\left(k_{2}=\Re\left(k_{2}^{*}\right)\right)$ in function of core-mantle boundary (CMB) radius, $R_{c}$, for the three selected compositional models (V1, circles; V4, squares; and V5, triangles; see text). Symbols with and without a black contour indicate which temperature profile is considered ( $T_{\text {hot }}$ and $T_{\text {cold }}$, respectively; see text for more details), and their color depicts the chosen viscosity of the mantle. Grey symbols represent the elastic case. Bars on $k_{2}$ represent the variation on the viscoelastic deformation related to the value of the rheological parameter $\alpha$ that describes the frequency dependence of the transient response in the Andrade model (see section 3.1). Dashed line represents the value of $k_{2}$ obtained by Konopliv and Yoder [1996] using Magellan and Pioneer data, the upper error bar being depicted by the long-dashed line. The lower estimate is too small to be drawn on this figure, due to the chosen scale.

models with or without the atmosphere indicate that the Love number is reduced by about $3-4 \%$ when the effect of the atmosphere is considered, depending on the assumed density and viscosity profile in the interior. This is comparable to the prediction of Dermott [1979], recently reevaluated by Remus et al. [2012], who showed that the overloading induced by a tidally deformed fluid envelope on the solid interior reduces the tidal response of this interior for Earth-sized planets having a ratio between the surface radius and the radius of the external envelope larger than 0.84 . In the case of Venus, this ratio, $R_{P} / R_{\text {atm }}$ is 0.984 , which corresponds to a reduction of interior response of about 3 to 3.5\% (according to Figure 5 of Remus et al. [2012] for Earth-sized planets), consistent with our computation.

\section{Predicted Venusian Love Numbers and Phase Shifts}

We compute the venusian deformation due to solar tides (period of 58.4 days) using the viscoelastic model described in section 3.1. We obtain the Love numbers and the dissipation factor for the six couples (compositional model/temperature profile) assuming a constant viscosity in the mantle (that can be related to the average viscosity; see section 3.2) of $10^{19}, 10^{20}, 10^{21}$, or $10^{22} \mathrm{~Pa}$ s. Results are displayed in Figures 3-5. As mentioned in section 3.3, values between 0.2 and 0.3 produce results for the Earth that are in good agreement with measurements. We therefore used this same range of value to compute the tidal deformation of Venus.

Figure 3 displays the potential Love number as a function of the core radius $R_{c}$ (see Table 2). We also computed the elastic deformation (grey symbols) to compare our results to Yoder [1995]'s ones. The large difference (up to more than $+20 \%$ on $k_{2}$ ) obtained when taking into account the viscosity of the mantle highlights the importance of the viscoelastic rheology for such a tidal period (58.4 days). Our results all fall into the range of uncertainty of the estimated potential Love number $\left(k_{2}=0.295 \pm 0.066\right)$ using Magellan and Pioneer Venus Orbiter spacecraft data [Konopliv and Yoder, 1996]. However, if a better accuracy (about 0.01) is obtained with a future mission to Venus, Figure 3 shows that a high value of $k_{2}(>0.34)$ will favor a hot and low viscous mantle (about $10^{19} \mathrm{~Pa} \mathrm{~s}$ ) and an Earth-like composition (V5) or a low FeO content (model V1). Consequently, a high $k_{2}$ value will reveal the existence of a large core $\left(R_{c}>3300 \mathrm{~km}\right)$. In the opposite case, a low $k_{2}$ value 


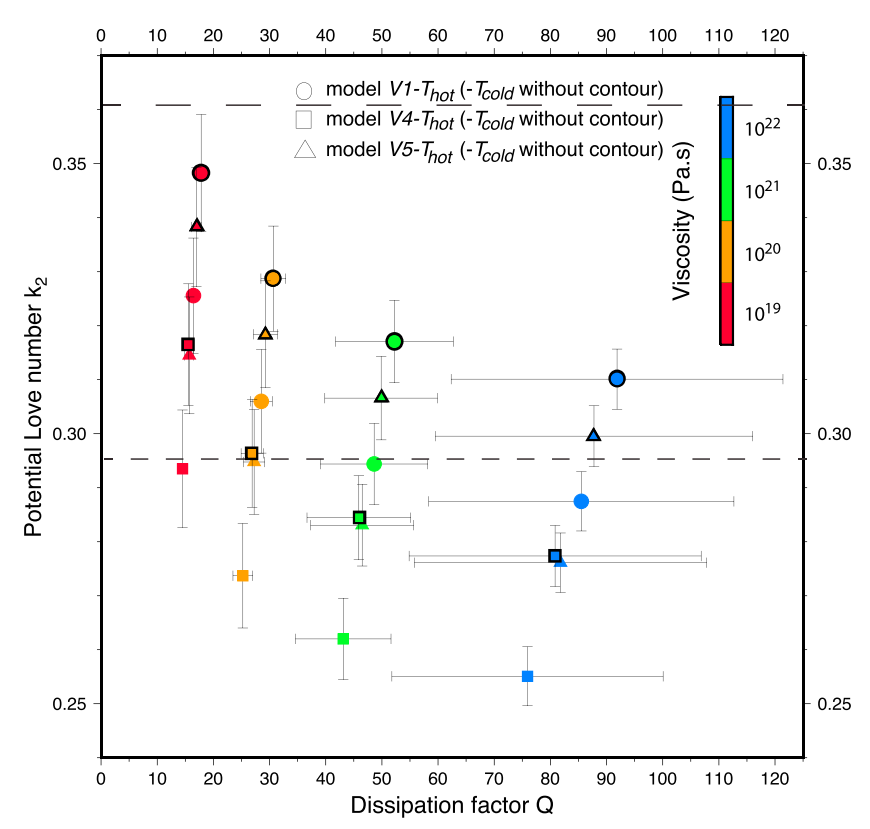

Figure 4. Potential Love number $k_{2}\left(k_{2}=\Re\left(k_{2}^{*}\right)\right)$ in function of the dissipation factor $Q$. Legend for symbols, colors, and lines are the same as in Figure 3.

$(<0.27)$ will be the signature of a colder and higher viscous mantle $\left(10^{21}-10^{22} \mathrm{~Pa} s\right)$ and of a high FeO content (model V4) which would indicate a smaller core $\left(R_{c}<3000 \mathrm{~km}\right)$. The uncertainty on $k_{2}$ (linked to the chosen value of the parameter $\alpha$ ) is larger for a low viscosity in the mantle (about 3.5\% for $10^{19} \mathrm{~Pa} \mathrm{~s}$ ), while it diminishes for higher viscosities (slightly smaller or equal to $2 \%$ for $10^{22} \mathrm{~Pa} \mathrm{~s}$ ). While the effect of varying the parameter $\alpha$ remains in any case small for the potential Love number, the uncertainty on the dissipation factor $Q$ can be large, depending on the mantle viscosity. Figure 4 shows that the uncertainty on $Q$ is the largest for a high viscosity in the mantle (about 30\% for $10^{22} \mathrm{~Pa} \mathrm{~s}$ ) and the smallest for a low viscosity (about $5 \%$ for $10^{19} \mathrm{~Pa} \mathrm{~s}$ ).

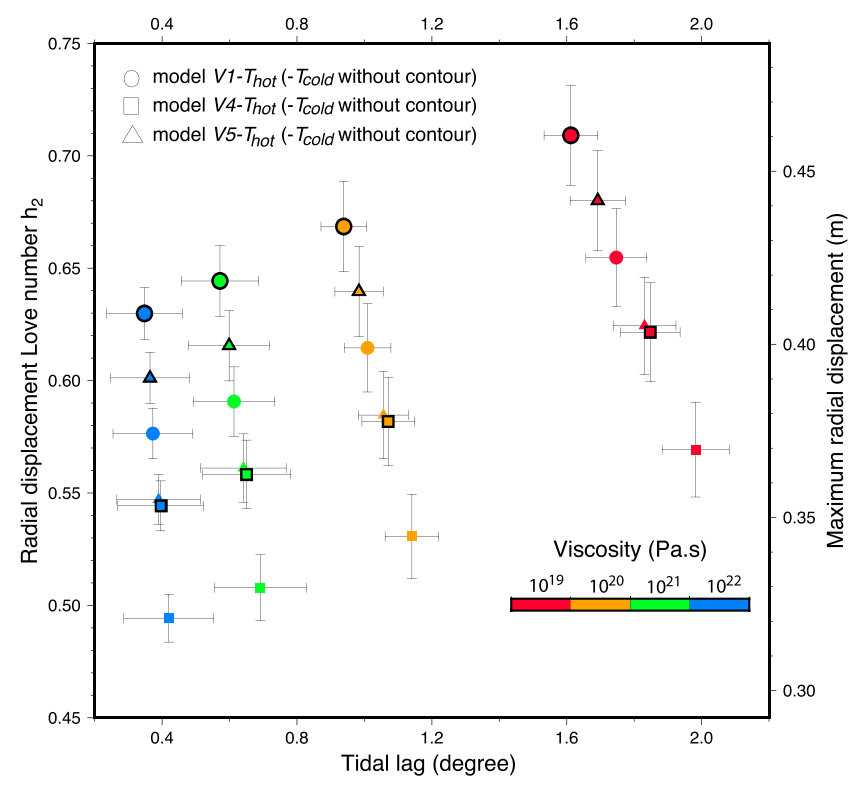

Figure 5. Radial displacement Love number $\left(h_{2}\right)$, together with its dimensioned value of maximum radial displacement in meters $\left(h_{\max }\right)$, in function of the $k_{2}$ tidal phase lag in degrees $(\epsilon)$. Note that the difference between the $k_{2}$ and $h_{2}$ lags is smaller than $2.9 \%$ whatever the chosen compositional model, temperature profile, and mantle viscosity. Legend for symbols, colors, and lines are the same as in Figure 3. 
The dissipation factor can also be expressed as the potential tidal phase lag ( $\epsilon$, in radian) following the relationship: $2 \epsilon=\arcsin Q^{-1} \simeq Q^{-1}$. Figure 5 displays the radial displacement Love number, $h_{2}$, in function of the tidal phase lag (in degrees). The right $y$ axis stands for the maximum radial displacement at the surface. It is related to $h_{2}$ with the following equation:

$$
h_{\max }=h_{2} \frac{3}{2} \frac{M_{S}}{M_{V}}\left(\frac{R_{p}}{d_{S}}\right)^{3} R_{P},
$$

$M_{S}$ and $d_{S}$ being the mass of the Sun and the Venus to Sun distance, respectively. This peak to peak amplitude of radial displacement at the equator varies between 30 and $50 \mathrm{~cm}$, depending on the models (mantle composition, temperature, and viscosity). An accuracy of $2 \mathrm{~cm}$ on the measure of the maximal radial displacement would allow the identification of the best compositional model. For low viscosities, the tide-induced phase lag lies between 1.6 and $2^{\circ}$ (it corresponds to a distance of 170 to $210 \mathrm{~km}$ at the equator), while it is smaller than $0.8^{\circ}$ for the largest viscosities (i.e., a distance smaller than $85 \mathrm{~km}$ at the equator). Therefore, a precise determination, from the orbit of a satellite, of the angle of the tidal bulge to the sun (by measuring the degree- 2 potential or the tidal displacement) would give crucial insight on the average viscosity in the Venusian mantle. Coupled with a more precise estimation of the potential Love number, it could yield further indications on the mantle composition.

\section{Constraining the State of the Core}

As mentioned in section 1, Yoder [1995] computes the elastic deformation of Venus due to tides for a suite of models parameterized by the molar fraction of $\mathrm{Mg}$ relative to $\mathrm{Fe}$ in the mantle and the radius of the liquid core. The difference in properties between magnesium and iron end-members in the olivine family were used to extrapolate PREM values to different Mg to Fe molar fractions, assuming that such difference is representative of the $\mathrm{Mg}$ to Fe ratio effect on the properties of the whole mantle, whatever the composition. One of the major results of this study was the strong decrease of the $k_{2}$ Love number for a completely solid iron core (of the order of 0.17 instead of a range between 0.23 and 0.29). One year later, Konopliv and Yoder [1996] estimated the potential Love number from Doppler tracking of Magellan and Pioneer Venus Orbiter to be $k_{2}=0.295 \pm 0.066$ and naturally concluded that Venus' core is liquid. However, there is no other data that can confirm this conclusion: the fact that there is no internal magnetic field can support the fact that Venus' core is liquid, without crystallization to enhance the chemically driven convection [Stevenson et al., 1983], but it can also support the fact that the core might be entirely solid, due to efficient thermal transfer in Venus' history [Arkani-Hamed and Toksöz, 1984]. A solid inner core might also coexist with a liquid outer core depending on the style of past mantle convection and therefore on its ability to extract heat from the core [Stevenson, 2003]. Indeed, recent models of thermal evolution indicate that a transition from dynamo to no dynamo seems likely, particularly in the episodic mode [Armann and Tackley, 2012]. The fact that there is no actual internal magnetic field indicates solely that the core is presently not sufficiently cooling to sustain a chemically driven dynamo [Stevenson, 2003]. Very recent progress has been made in the knowledge of the phase diagrams of Fe, considering or not light elements [e.g., Zhang et al., 2016; Dorogokupets et al., 2017]. However, the state of the core cannot be directly derived from these studies as the pressure and temperature at the core-mantle boundary $(\mathrm{CMB})$ are strongly uncertain. Indeed, the pressure at the $\mathrm{CMB}$ will depend on the radius of the core and therefore on the compositional model and on the mantle temperatures (see Table 2). Our choice of considering five compositional models associated with two end-member temperature profiles echoes these uncertainties. The temperature drop at the CMB may also be questioned. In the Earth, the temperature drop across $\mathrm{D}^{\prime \prime}$ is estimated using experimental measurements of phase transitions such as perovskite/postperovskite located close to the CMB thanks to the use of the seismic velocities. For Venus, the temperature drop and the thickness of the hot thermal boundary layer reported in Steinberger et al. [2010] and in Aitta [2012] are directly rescaled from the Earth's value. However, the thermal history of the planet might have a large influence of the thermal state of its core. In our view, the uncertainties on the core composition, the temperature at the CMB, and the thermal history of Venus are sufficiently large to not rule out a priori the existence of a solid core.

In consequence, we reinvestigate the influence of the state of the core on the viscoelastic deformation, while Yoder [1995] used a purely elastic rheology. We consider (as an end-member) the case of a solid core composed of pure iron. The density variations with pressure of hcp-Fe along an isentropic temperature profile given in Dorogokupets et al. [2017] are used. We use the compositional model V5 and the "hot" temperature profile in 


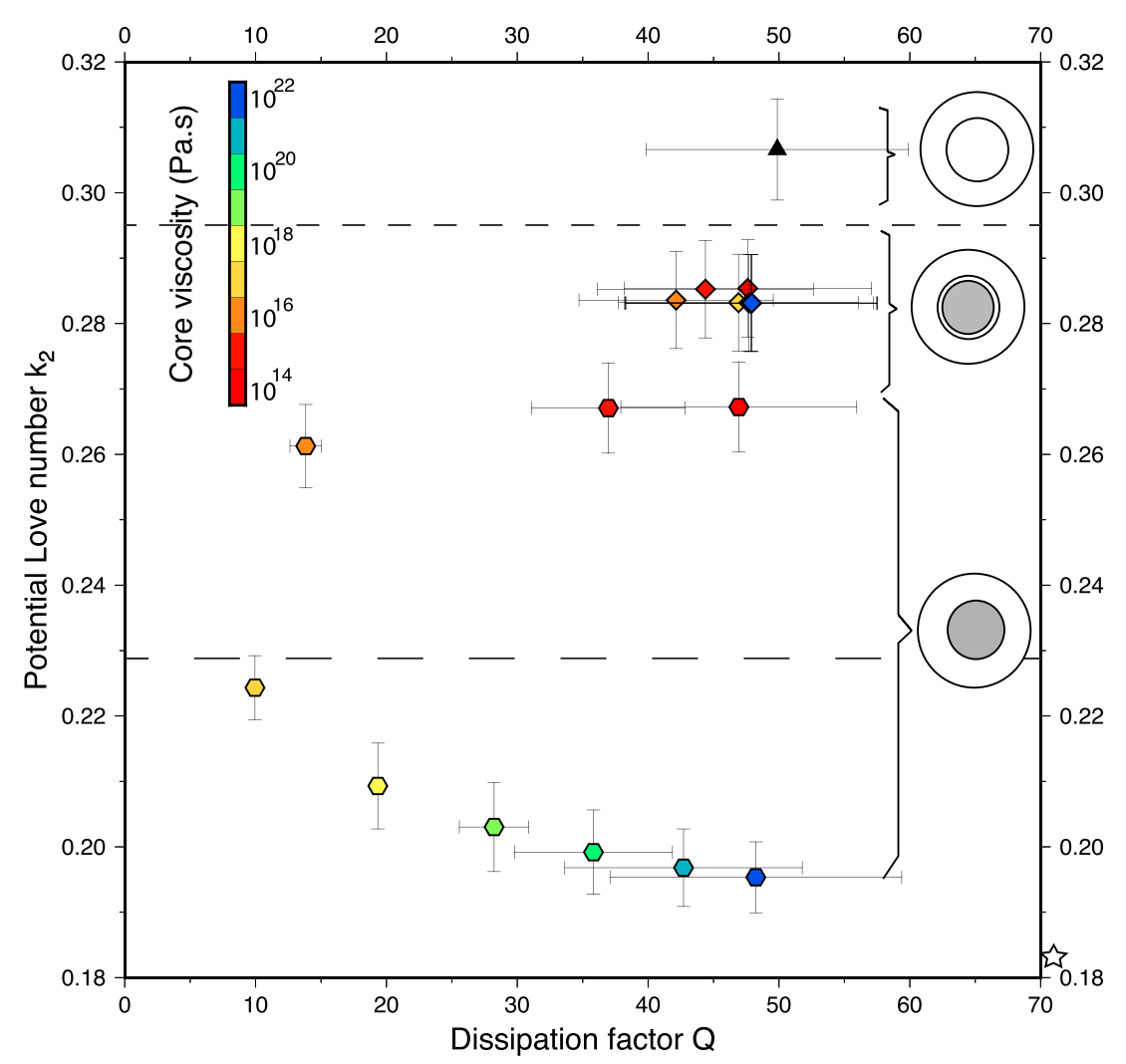

Figure 6. Potential Love number, $k_{2}$, in function of the dissipation factor $Q$ using the compositional model V5, the "hot" temperature profile, and a mantle viscosity of $10^{21} \mathrm{~Pa}$ s. The case of a solid core of pure iron is illustrated using hexagons, and the case of a $2500 \mathrm{~km}$ radius solid core and a liquid outer core with diamonds. The black triangle stands for the case with an entirely liquid core, corresponding to the green triangle with black contour in Figure 4, for comparison. Colors of hexagons depict the chosen viscosity of the solid core. The star on the right side shows the result obtained for the elastic solid core case for comparison with Yoder [1995]'s study $\left(k_{2}=0.1836\right)$. The long-dashed line represents the lowest estimate of $k_{2}$ from Konopliv and Yoder [1996], the upper one being too large to be drawn on the figure due to the chosen scale.

the mantle. This new model obviously differs from the previous V5- $T_{\text {hot }}$ model: the density of the pure iron core is larger than the previous density derived from PREM, as the Earth's core contains light elements. The size of the core is then smaller $(3074 \mathrm{~km}$ instead of $3292 \mathrm{~km})$, and pressure and temperature at the CMB are equal to $120 \mathrm{GPa}$ and $3900 \mathrm{~K}$, which is at the limit of the solid domain. The seismic velocities in the solid core are derived from their evolution in the Earth's inner core (given by the PREM model) rescaled with the pure iron density. Concerning the viscosity of the Earth's inner core, published estimations go from $10^{11}$ to $10^{22} \mathrm{~Pa} s$ (using either experiments [Gleason and Mao, 2013], observations [e.g., Koot and Dumberry, 2011] or creep mechanisms [e.g., Orman, 2004; Reaman et al., 2011]). We investigate a large range of core viscosity from $10^{14}$ to $10^{22} \mathrm{~Pa}$ s, since this parameter has a large influence on the viscoelastic deformation. Figure 6 displays the potential Love number as a function of the dissipation factor, together with their variation linked to the value of the parameter $\alpha$ (see section 4).

We also performed an intermediate case with a solid inner core of $2500 \mathrm{~km}$ radius composed of pure iron and a liquid outer core enriched in light elements (density variations are extrapolated from the Earth's outer core using PREM). As expected, the core radius is then intermediate, equal to $3183 \mathrm{~km}$. As depicted by the diamonds on Figure 6, the influence of the viscosity of the core is strongly reduced in the case of a solid core surrounded by a liquid outer core. The difference in the tidal deformation with the case of a planet having a completely liquid core is then mainly due to the size of the core.

We can conclude from the behavior of the viscoelastic deformation when including a solid core in our models that, if the $k_{2}$ Love number is smaller than 0.25 , the core should be solid with a high viscosity (above $10^{17} \mathrm{~Pa} \mathrm{~s}$ ). On the other hand, if the tidal phase lag $\epsilon$ is large (above $2^{\circ}$ ), the core should also be solid with a viscosity of 
the order of $10^{16-17} \mathrm{~Pa}$ s, whatever the value of $k_{2}$. In the other cases $\left(k_{2}>0.25\right.$ and $\left.\epsilon<2^{\circ}\right)$, the core might be entirely liquid, entirely solid with a low viscosity (less than $10^{16} \mathrm{~Pa} s$ ), or partly liquid with a solid inner core. Our calculations indicate that due to large uncertainties on the $k_{2}$ Love number [Konopliv and Yoder, 1996], the possibility of a solid core inside Venus cannot be ruled out when viscoelasticity is taken into account.

\section{Implications for Future Missions to Venus}

The $k_{2}$ tidal Love number expresses the modification of the gravitational potential of the planet due to its tidal deformation. This small gravitational modification is sensed by spacecraft orbiting the planet and can be detected as fine perturbations of the orbital velocity monitored from tracking data of the spacecraft (Doppler tracking performed with Earth-based large antennas [e.g., Holmes et al., 2008]. This method of perturbations is, however, challenging since it requires to properly separate all sources of velocity perturbations related to the $k_{2}$ tidal Love number and the gravitational field from other nongravitational forces like those due to the attitude maneuvers and to the drag in the upper atmosphere at the lowest altitude part of the spacecraft orbit [e.g., Rosenblatt and Dehant, 2010]. This is the main limitation of the solution of $k_{2}$ determined from the Magellan tracking data [Konopliv and Yoder, 1996]. The accuracy on the $k_{2}$ solution is also affected by the error on the gravity field solution. The current solution from Magellan tracking data has an accuracy that is not uniform at the surface of the planet. The spatial resolution is as worse as the degree 40 (about $475 \mathrm{~km}$ of spatial resolution) in large areas of both northern and southern hemispheres and as good as the degree 100 (200 km of spatial resolution) in the equatorial band [Konopliv et al., 1999]. This is due to the orbits chosen during cycles 4 (very elliptical) and 5 and 6 (less elliptical).

The EnVision candidate mission to ESA's M5 [Ghail et al., 2016] call plans to have a spacecraft with a polar and circular orbit at an altitude of about $250 \mathrm{~km}$. Such an orbit will allow avoiding drag effects on the reconstructed orbit. A better a priori knowledge of the maneuvers than for Magellan spacecraft [Konopliv and Yoder, 1996] is also expected as for current Martian spacecraft. The residual accelerations due to the attitude maneuvers are now known at an average level of $0.003 \mathrm{~mm} / \mathrm{s}$ (with fluctuations as worse as $0.05 \mathrm{~mm} / \mathrm{s}$ ), like for Mars Express, [Rosenblatt et al., 2008], against $0.5 \mathrm{~mm} / \mathrm{s}$ at the time of the Magellan mission [Konopliv et al., 1999]. It should improve the knowledge of the gravity field by uniformizing its spatial resolution up to degree 120-150 (about $125-150 \mathrm{~km}$ of spatial resolution).

We have assessed the accuracy on the $k_{2}$ solution that can be reached using tracking data of these candidate missions. We have first simulated $X$ band Doppler tracking data on the basis of a nominal scenario with $8 \mathrm{~h}$ of tracking per day over about 1000 successive days. We have added a conservative noise of $0.05 \mathrm{~mm} / \mathrm{s}$ for $10 \mathrm{~s}$ Doppler count time. Finally, we have used the current $k_{2}$ value to simulate these noisy tracking data. Then, using the classical orbit determination procedure, we have assessed the retrieval of the $k_{2}$ value from these simulated tracking data (i.e., least squares fit of initial state vector and parameters of the model of the spacecraft motion to Doppler tracking data) [e.g., Rosenblatt et al., 2008; Konopliv et al., 2016; Genova et al., 2016]. Here we used the GINS software to perform our simulations (this software is fully described in the documentation available at http://grgs.obs-mip.fr/content/download/1460/9226/file/GINS_Algo_2013.pdf). We have obtained an accuracy better than $1 \%$, which is largely optimistic with respect to the current accuracy of $20 \%$ [Konopliv and Yoder, 1996]. This optimistic solution is obviously due to the fact that we did not take into account the inaccuracies on the force model (maneuver, drag, and gravity field) of the spacecraft motion in our simulations. We have performed additional simulations by adding the effect of the error on the gravity field. First results seem to indicate that the error on the gravity field should be improved by a factor of 10 to 20 from tracking data of EnVision mission in order to reach an error of 0.01 (or about 3\%) on the $k_{2}$ solution as required to improve our knowledge of Venus' interior. Such a required accuracy should correspond to an error at the meter level on the reconstructed orbit, which seems to be very challenging although it corresponds to the current accuracy of the Martian spacecraft orbit that have allowed a $k_{2}$ Love number solution with a current error of about 3\% [e.g., Konopliv et al., 2016; Genova et al., 2016]. However, our simulations need further developments, to take the maneuver effects on orbit reconstruction into account, which are out of the scope of this paper.

The state of the core is a crucial question to understand Venus' thermal evolution. But, as shown above, our knowledge of the potential Love number is not sufficiently accurate to infer the state of the core. Rotation measurements, such as nutations observations (that can be used to constrain Mars' and Earth's deep interior [e.g., Van Hoolst, 2015]), usually offer an opportunity to sound the state of the core but are not supposed to 
be useful in Venus' case because resonance effects with the free core nutation are expected to be negligible [Van Hoolst, 2015]. As for Martian spacecrafts, the EnVision spacecraft would also offer the opportunity to improve our knowledge of the proper motion of the planet (precession rate and the length of day, LOD, variations). These parameters are expected to have a signature in the orbital perturbations of the spacecraft. The combination with the radar interferometry techniques might improve our knowledge of the precession rate and LOD variation amplitudes. These orientation and rotation variations of Venus are related to the internal mass distribution and might thus improve our interior models [Cottereau et al., 2011]. However, in the absence of restrictive observations, coupled models of dynamics and thermal evolution of the mantle and the core, together with experimental measurements of iron alloys phase diagrams should be best used to possibly distinguish a liquid from a solid core.

\section{Conclusion}

We performed computation of tidal viscoelastic deformation of Venus using six different models of internal structure, corresponding to three different formation scenarii (two end-members in terms of core radius and an Earth-like model) and to two end-member temperature profiles in the mantle. The density and seismic velocity profiles for each models are computed using the Perple_X program in the mantle and a PREM extrapolation in the core. A large range of mantle viscosity has been tested ( $10^{19}$ to $10^{22} \mathrm{~Pa}$ s), as well as the state of the core (solid/liquid). We show that the anelasticity of the mantle significantly increases the potential Love number, $k_{2}$, relative to the elastic models [Yoder, 1995]: depending on the viscosity of the mantle, up to $+25 \%$ for a liquid core and up to $89 \%$ in the case of a low ( $\leqslant 10^{16} \mathrm{~Pa} \mathrm{~s}$ ) viscosity solid core. Due to these anelasticity effects, we show that the possibility of a completely solid metal core inside Venus cannot be ruled out based on the estimate of $k_{2}$ comprised between 0.23 and 0.36 from the Magellan mission [Konopliv and Yoder, 1996]. Our calculations show that for a solid core of pure iron, a potential Love number as high as $0.265-0.27$ can be obtained, within the error bars of the current estimate, if the core viscosity is lower than $10^{17} \mathrm{~Pa}$ s. A solid core could be ruled out only if its viscosity is higher than $10^{17} \mathrm{~Pa}$ s. A Love number lower than or equal to 0.26 associated with a large potential tide-induced phase lag $\left(>2^{\circ}\right)$ would be a clear indication of a solid iron core. This would imply that the core contained no or very few light elements and that the mantle has been very efficient at cooling down the interior over the age of the solar system, possibly associated with episodic mantle overturn [Armann and Tackley, 2012]. For smaller tidal phase lags $\left(<1.5^{\circ}\right)$, there would still be ambiguity between a fully solid core and a partially or fully liquid core with a high mantle viscosity ( $\geq 10^{22} \mathrm{~Pa} s$ ).

Potential Love numbers higher than $0.27-0.28$ would indicate that the core is partially or entirely liquid, and the precise determination of the Love numbers, $k_{2}$ and $h_{2}$, combined with an estimate of the tidal phase lag can be used to infer the composition, size, and viscosity of the mantle. The monitoring of the tidal deformation will allow the definition of a class of interior models. As an example, a small value (less than $0.8^{\circ}$ ) would indicate a highly viscous mantle $\left(10^{21} \mathrm{~Pa}\right.$ s at least), while a large angle (from 1.6 to $2^{\circ}$ ) would be the signature of a low mantle viscosity (of the order of $10^{19} \mathrm{Pas}$ ). This kind of information is crucial to constrain the thermal evolution of Venus' interior and to better understand the link with the evolution of its thick $\mathrm{CO}_{2}$ atmosphere [e.g., Gillmann and Tackley, 2014].

A precise determination of the potential Love number (with an error of 0.01 ) associated with a precise measurement of the maximum radial displacement and of the tide-induced phase lag, is a challenge for the future exploration of Venus. The EnVision mission project under consideration by ESA [Ghail et al., 2016] has the capability to significantly improve the tidal measurements compared to the Magellan mission. However, detailed simulations of orbit reconstruction are required to determine precisely the accuracy that could be reached. Such measurements from the orbit once combined with tidal deformation computations, such as those presented here, and thermal evolution models, will provide crucial information on the thermal history of Venus and will help understanding why its evolution diverges from its twin sister, the Earth.

\section{References}

Aitta, A. (2012), Venus' internal structure, temperature and core composition, Icarus, 218, 967-974. Anderson, D. L. (1989), Theory of the Earth, 366 pp., Blackwell Scientific Publ., Oxford.

Arkani-Hamed, J., and M. N. Toksöz (1984), Thermal evolution of venus, Phys. Earth Planet. Int., 34, 232-250.

Armann, M., and P. J. Tackley (2012), Simulating the thermochemical magmatic and tectonic evolution of Venus's mantle and lithosphere: Two-dimensional models, J. Geophys. Res., 117, E12003, doi:10.1029/2012JE004231.

Basaltic Volcanism Study Project (1981), Basaltic Volcanism on the Terrestrial Planets, Pergamon Press, New York. 
Benjamin, D., J. Wahr, R. D. Ray, G. D. Egbert, and S. D. Desai (2006), Constraints on mantle anelasticity from geodetic observations, and implications for the $\mathrm{J}_{2}$ anomaly, Geophys. J. Int., 165, 3-16, doi:10.1111/j.1365-246X.2006.02915.x.

Castillo-Rogez, J. C., M. Efroimsky, and V. Lainey (2011), The tidal history of lapetus: Spin dynamics in the light of a refined dissipation model, J. Geophys. Res., 116, E09008, doi:10.1029/2010JE003664.

Čižková, H., A. P. van den Berg, W. Spakman, and C. Matyska (2012), The viscosity of earth's lower mantle inferred from sinking speed of subducted lithosphere, Phys. Earth Planet. Int., 200-201, 56-62.

Connolly, J. A. D. (2005), Computation of phase equilibria by linear programming: A tool for geodynamic modeling and its application to subduction zone decarbonation, Earth Planet. Sci. Lett., 236, 524-541, doi:10.1016/j.epsl.2005.04.033.

Cottereau, L., N. Rambaux, S. Lebonnois, and J. Souchay (2011), The various contributions in venus rotation rate and lod, Astron. Astrophys., $531, \mathrm{~A} 45$.

Dermott, S. F. (1979), Tidal dissipation in the solid cores of the major planets, Icarus, 37, 310-321, doi:10.1016/0019-1035(79)90137-4.

Dorogokupets, P. I., A. M. Dymshits, K. D. Litasov, and T. S. Sokolova (2017), Thermodynamics and equations of state of iron to 350 gpa and 6000 k, Sci. Rep., 7, 41863.

Dziewonski, A. M., and D. L. Anderson (1981), Preliminary reference Earth model, Phys. Earth Planet. Int., 25, 297-356, doi:10.1016/0031-9201(81)90046-7.

Fegley, B. J. (2014), Venus, Treatise on Geochemistry, vol. 2, chap. 2.7, pp. 127-148, Elsevier Science, Amsterdam.

Furuya, M., and B. F. Chao (1996), Estimation of period and Q of the Chandler wobble, Geophys. J. Int., 127, 693-702, doi:10.1111/j.1365-246X.1996.tb04047.x.

Genova, A., S. Goossens, F. Lemoine, E. Mazarico, G. Neumann, D. Smith, and M. T. Zuber (2016), Seasonal and static gravity field of mars from mgs, mars odyssey and mro radio science, Icarus, 272, 228-245.

Ghail, R., W. F. Colin, and T. Widemann (2016), Envision M5 venus orbiter proposal: Opportunities and challenges, in Proceedings of the 47th Lunar and Planetary Science Conference, pp. 1511, LPI Contribution No. 1903, The Woodlands, Tex.

Gillmann, C., and P. Tackley (2014), Atmosphere/mantle coupling and feedbacks on venus, J. Geophys. Res. Planets, 19, 1189-1217.

Gleason, A. E., and W. L. Mao (2013), Strength of iron at core pressures and evidence for a weak Earth's inner core, Nat. Geosci., 6, 571-574.

Goettel, K. A., J. A. Shields, and D. A. Decker (1981), Density constraints on the composition of venus, Proc. Lunar Planet. Sci., 12B, $1507-1516$.

Holmes, D., et al. (2008), The challenges and opportunities for international cooperative radio science; experience with the mars express and venus express missions, in Proceedings of the AIAA/AAS Astrodynamics Specialist Conference, 18 pp., American Inst. of Aeronautics and Astronautics 2008-3556, Reston, Va.

Irifune, T. (1987), An experimental investigation of the pyroxene-garnet transformation in a pyrolite composition and its bearing on the constitution of the mantle, Phys. Earth Planet. Int., 45, 324-336, doi:10.1016/0031-9201(87)90040-9.

Jackson, I., J. D. Fitz Gerald, U. H. Faul, and B. H. Tan (2002), Grain-size-sensitive seismic wave attenuation in polycrystalline olivine, J. Geophys. Res., 107, 2360, doi:10.1029/2001JB001225.

Karato, S.-I. (2008), Deformation of Earth Materials, Cambridge Univ. Press, New York.

Karato, S. I., and P. Wu (1993), Rheology of the upper mantle: A synthesis, Science, 260, 771-778.

Konopliv, A., and C. Yoder (1996), Venusian k2 tidal love number from magellan and pvo tracking data, Geophys. Res. Lett., 23, 1857-1860.

Konopliv, A., R. Park, and W. M. Folkner (2016), An improved JPL Mars gravity field and orientation from Mars orbiter and lander tracking data, Icarus, 274, 253-260.

Konopliv, A. S., W. B. Banerdt, and W. L. Sjogren (1999), Venus gravity: 180th degree and order model, Icarus, 139, 3-18.

Koot, L., and M. Dumberry (2011), Viscosity of the Earth's inner core: Constraints from nutation observations, Earth Planet. Sci. Lett., 308(3-4), 343-349.

Korenaga, J. (2005), Firm mantle plumes and the nature of the core-mantle boundary region, Earth Planet. Sci. Lett., 232(1-2), 29-37.

Lewis, J. S. (1972), Metal/silicate fractionation in the solar system, Earth Planet. Sci. Lett., 15, 286-290, doi:10.1016/0012-821X(72)90174-4.

Mitrovica, J. X., and A. M. Forte (2004), A new inference of mantle viscosity based upon joint inversion of convection and glacial isostatic adjustment data, Earth Planet. Sci. Lett., 225, 177-189.

Mocquet, A., P. Rosenblatt, V. Dehant, and O. Verhoeven (2011), The deep interior of Venus, Mars, and the Earth: A brief review and the need for planetary surface-based measurements, Planet. Space Sc., 59, 1048-1061, doi:10.1016/j.pss.2010.02.002.

Morard, G., D. Andrault, D. Antonangeli, and J. Bouchet (2014), Properties of iron alloys under the Earth's core conditions, C. R. Geosci., 346, 130-139, doi:10.1016/j.crte.2014.04.007.

Morgan, J. W., and E. Anders (1980), Chemical Composition of Earth, Venus, and Mercury, Proc. Natl. Acad. Sci. U.S.A., 77, 6973-6977, doi:10.1073/pnas.77.12.6973.

Noack, L., D. Breuer, and T. Spohn (2012), Coupling the atmosphere with interior dynamics: Implications for the resurfacing of venus, Icarus, 217, 484-498.

Orman, J. V. (2004), On the viscosity and creep mechanism of Earth's inner core, Geophys. Res. Lett., 31, L20606, doi:10.1029/2004GL021209.

Pauer, M., K. Fleming, and O. Čadek (2006), Modeling the dynamic component of the geoid and topography of Venus, J. Geophys. Res., 111 , E11012, doi:10.1029/2005JE002511.

Phillips, R. J., M. A. Bullock, and S. A. Hauck II (2001), Climate and interior coupled evolution on Venus, Geophys. Res. Lett., 28(9), 1779-1782.

Ray, R. D., and G. D. Egbert (2012), Fortnightly earth rotation, ocean tides and mantle anelasticity, Geophys. J. Int., 189, 400-413.

Ray, R. D., R. J. Eanes, and F. G. Lemoine (2001), Constraints on energy dissipation in the earth's body tide from satellite tracking and altimetry, Geophys. J. Int., 144, 471-480.

Reaman, D. M., G. S. Daehn, and W. Panero (2011), Predictive mechanism for anisotropy development in the Earth's inner core, Earth Planet. Sci. Lett., 312(3-4), 437-442.

Remus, F., S. Mathis, J.-P. Zahn, and V. Lainey (2012), Anelastic tidal dissipation in multi-layer planets, Astron. Astrophys., 541, A165, doi:10.1051/0004-6361/201118595.

Richards, M., and B. Hager (1984), Geoid anomalies in a dynamic Earth, J. Geophys. Res., 89, 5987-6002.

Ringwood, A. E. (1977), Composition and Origin of the Earth, 65 pp., Res. School of Earth Sciences Publ. No 1227, Australian National Univ., Canberra.

Rosenblatt, P., and V. Dehant (2010), Mars geodesy, rotation and gravity, Res. Astron. Astrophys., 10(8), 713-736.

Rosenblatt, P., V. Lainey, S. Le Maistre, J. Marty, V. Dehant, M. Pätzold, T. Van Hoolst, and B. Häusler (2008), Accurate mars express orbits to improve the determination of the mass and ephemeris of the martian Moons, Planet. Space Sci., 56, 1043-1053.

Rozel, A. (2012), Impact of grain size on the convection of terrestrial planets, Geochem. Geophys. Geosyst., 13, Q10020, doi:10.1029/2012GC004282.

Saito, M. (1974), Some problems of static deformation of the Earth, J. Phys. Earth, 22, 123-140. 
Sohl, F., and G. Schubert (2015), Interior structure, composition, and mineralogy of the interior structure, composition, and mineralogy of the terrestrial planets, Treat. Geophys., 10, 23-64.

Solomatov, S., and C. C. Reese (2008), Grain size variations in the Earth's mantle and the evolution of primordial chemical heterogeneities, J. Geophys. Res., 113, B07408, doi:10.1029/2007JB005319.

Stacey, F. D., and P. M. Davis (1998), Physics of the Earth, 4th edn., 548 pp., Cambridge Univ. Press, Cambridge, U. K.

Steiff, A., J. T. Schofield, A. J. Kliore, F. W. Taylor, S. S. Limaye, H. E. Revercomb, L. A. Sromovsky, V. V. Kerzhanovich, V. I. Moroz, and M. Y. Marov (1985), Models of the structure of the atmosphere of venus from the surface to 100 kilometers altitude, Adv. Space Res., 5(11), 3-58.

Steinberger, B., S. C. Werner, and T. H. Torsvik (2010), Deep versus shallow origin of gravity anomalies, topography and volcanism on Earth, Venus and Mars, Icarus, 207, 564-577, doi:10.1016/j.icarus.2009.12.025.

Stevenson, D. J. (2003), Planetary magnetic fields, Earth Planet. Sci. Lett., 208, 1 - 11.

Stevenson, D. J., T. Spohn, and G. Schubert (1983), Magnetism and ther-mal evolution of the terrestrial planets, Icarus, 54, 466-489.

Stixrude, L., and C. Lithgow-Bertelloni (2011), Thermodynamics of mantle minerals - II. Phase equilibria, Geophys. J. Int., 184, 1180-1213, doi:10.1111/j.1365-246X.2010.04890.x.

Takeushi, H., and M. Saito (1972), Seismic surfaces waves, in Methods in Computational Physics, vol. 1, edited by B. A. Bolt, pp. 217-295, Acad. Press, New York.

Tan, B. H., I. Jackson, and J. D. Fitz Gerald (2001), High-temperature viscoelasticity of fine-grained polycrystalline olivine, Phys. Chem. Miner., $28,641-664$.

Tobie, G., A. Mocquet, and C. Sotin (2005), Tidal dissipation within large icy satellites: Applications to Europa and Titan, Icarus, 177, 534-549, doi:10.1016/j.icarus.2005.04.006.

Van Hoolst, T. (2015), Rotation of the terrestrial planets, Treat. Geophys., 10, 121-151.

Weidenschilling, S. J. (1976), Accretion of the terrestrial planets. II, Icarus, 27, 161-170, doi:10.1016/0019-1035(76)90193-7.

Yoder, C. (1995), Venus' free obliquity, Icarus, 117, 250-286.

Zhang, Y., T. Sekine, H. He, Y. Yu, F. Liu, and M. Zhang (2016), Experimental constraints on light elements in the Earth's outer core, Sci. Rep., 6 , 22473.

Zharkov, V. N. (1983), Models of the internal structure of venus, The Moon and the Planets, 29, 139-175. 